\title{
Optimizing Combinations of Flavonoids Deriving from Astragali Radix in Activating the Regulatory Element of Erythropoietin by a Feedback System Control Scheme
}

\author{
Hui Yu, ${ }^{1,2}$ Wendy L. Zhang, ${ }^{3}$ Xianting Ding, ${ }^{4}$ Ken Y. Z. Zheng, \\ Chih-Ming Ho, ${ }^{5}$ Karl W. K. Tsim, ${ }^{3}$ and Yi-Kuen Lee ${ }^{2}$ \\ ${ }^{1}$ State Key Laboratory of Analytical Chemistry for Life Science, Nanjing University, Nanjing, Jiangsu, China \\ ${ }^{2}$ Department of Mechanical Engineering, The Hong Kong University of Science and Technology, Clear Water Bay Road, Hong Kong \\ ${ }^{3}$ Division of Life Science and Center for Chinese Medicine, The Hong Kong University of Science and Technology, \\ Clear Water Bay Road, Hong Kong \\ ${ }^{4}$ School of Biomedical Engineering, Med-X Research Institute, Shanghai Jiao Tong University, Shanghai, China \\ ${ }^{5}$ Center for Cell Control, Mechanical and Aerospace Engineering Department, Biomedical Engineering Department, \\ University of California, Los Angeles, CA 90095-1597, USA
}

Correspondence should be addressed to Karl W. K. Tsim; botsim@ust.hk and Yi-Kuen Lee; meyklee@ust.hk

Received 4 February 2013; Accepted 22 March 2013

Academic Editor: Shao Li

Copyright (C) 2013 Hui Yu et al. This is an open access article distributed under the Creative Commons Attribution License, which permits unrestricted use, distribution, and reproduction in any medium, provided the original work is properly cited.

\begin{abstract}
Identifying potent drug combination from a herbal mixture is usually quite challenging, due to a large number of possible trials. Using an engineering approach of the feedback system control (FSC) scheme, we identified the potential best combinations of four flavonoids, including formononetin, ononin, calycosin, and calycosin-7-O- $\beta$-D-glucoside deriving from Astragali Radix (AR; Huangqi), which provided the best biological action at minimal doses. Out of more than one thousand possible combinations, only tens of trials were required to optimize the flavonoid combinations that stimulated a maximal transcriptional activity of hypoxia response element (HRE), a critical regulator for erythropoietin (EPO) transcription, in cultured human embryonic kidney fibroblast (HEK293T). By using FSC scheme, 90\% of the work and time can be saved, and the optimized flavonoid combinations increased the HRE mediated transcriptional activity by $\sim 3$-fold as compared with individual flavonoid, while the amount of flavonoids was reduced by $\sim 10$-fold. Our study suggests that the optimized combination of flavonoids may have strong effect in activating the regulatory element of erythropoietin at very low dosage, which may be used as new source of natural hematopoietic agent. The present work also indicates that the FSC scheme is able to serve as an efficient and model-free approach to optimize the drug combination of different ingredients within a herbal decoction.
\end{abstract}

\section{Introduction}

Traditional Chinese medicine (TCM) has played an important role in primary health care in China of over thousands of years [1]. In contrast to isolated, bioactive, single natural products in Western medicine, TCM uses a mixture of active ingredients; this represents a holistic approach in disease prevention. TCM has attracted a lot of attention for serving as complementary health food supplements with low toxicity and fewer complications $[2,3]$. Astragali Radix (AR;
Huangqi), the dried root of Astragalus membranaceus (Fisch.) Bunge or A. membranaceus (Fisch.) Bunge var. mongholicus (Bunge) P. K. Hsiao, is one of the most widely used Chinese herbs as a health food supplement to reinforce "Qi" (vital energy) [4]. Pharmacological study has demonstrated that the water extract of AR possesses many biological functions, including hepatoprotective effects, neuroprotective effects against ischemic brain injury, hematopoietic, antioxidative, antihypertensive, immunological properties, cardiotonic, and antiaging activities $[5,6]$. 
Previous study also showed that the AR extract could improve hematopoietic functions by regulating erythropoietin (EPO) expression. EPO is an erythrocyte-specific hematopoietic growth factor produced by kidney and liver [7]. Failure to increase the amount of circulating EPO under hypoxia stress can lead to anemia [8]. Hypoxia response element (HRE), a critical regulator for EPO transcription, is located on the promoter region of the EPO gene. Under hypoxia condition, the activated hypoxia-induced factor (HIF) binds onto HRE and subsequently initiates EPO gene expression [9]. The AR regulating EPO expression was through an induction of the transcriptional activity of HRE [10]. One of major components in AR was flavonoid, for example, formononetin, ononin, calycosin, and calycosin7-O- $\beta$-D-glucoside. These four flavonoids can induce the expression of EPO [11]; however, the effect of a combination of these flavonoids has not been revealed. Indeed, the combined mixture is mimicking partly the scenario of a herbal mixture.

A major problem in combinatorial therapies lies in the number of possible combinations $[12,13]$, which becomes more challenging in optimizing Chinese herbal mixtures. Besides, the combined effect of numerous constituents within a herbal composite prescription is hard to validate [14]. Previous study indicated that different experimental methods have been used in discovering combinatorial therapies. Li et al. established an algorithm termed NIMS (Network TargetBased Identification of Multicomponent Synergy) to prioritize synergistic agent combinations in a high throughput way [15]. Both the topology score and agent score were proposed for the evaluation of agent interactions; Yan et al. developed a systematic simplification framework for drug combination design by combining simulation and system reaction network topology analysis [16]. Among different classes of strategy, Wong et al. developed a feedback system control (FSC) scheme to implement an iterative stochastic search [17]. FSC efficiently discovered potent combinations for inhibiting virus infection of fibroblasts, in only tens of iterations out of one hundred thousand possible trials. Recently, Tsutsui et al. further extended the FSC for parallel searching and identified a unique combination of three combined inhibitors that enables the maintenance of human embryonic stem cells [18]. Here, we aimed to optimize a herbal mixture for therapeutic goals by using the FSC scheme, and therefore the optimization of AR-derived flavonoid combinations was evaluated as an initial example. Having the FSC, we were able to quickly pinpoint the optimal flavonoid combinations for maximizing the HRE-mediated transcriptional activity.

\section{Materials and Methods}

2.1. Plant Materials and Chemicals. Three-year-old AR, the dry roots of $A$. membranaceus var. mongholicus, was collected from Shanxi province [4]. The authentication of plant materials was performed morphologically by Dr. Tina Dong of The Hong Kong University of Science and Technology (HKUST) during the field collection. The corresponding voucher no. 02-10-4, as forms of the whole plant were deposited in Center for Chinese Medicine, HKUST. Formononetin, calycosin, ononin, and calycosin-7-O- $\beta$-D-glucoside were purchased from Weikeqi Biotechnology Co. (Sichuan, China). The purities of these marker chemicals, confirmed by highperformance liquid chromatography (HPLC), were higher than $98.0 \%$. Analytical- and HPLC-grade reagents were from Merck (Darmstadt, Germany).

2.2. Preparation of AR Extracts and Chemicals. Dry roots of AR (50 g) were extracted twice with distilled water $(400 \mathrm{~mL})$ at $100^{\circ} \mathrm{C}$ for 2 hours. The extract was centrifuged at 3,000 $\mathrm{g}$ for $10 \mathrm{~min}$. The supernatant was freeze-dried (yield $=14.56 \mathrm{~g}$ ) and kept at $-20^{\circ} \mathrm{C}$. For standardization of AR extract, an Agilent 1200 series system (Agilent, Waldbronn, Germany), equipped with a degasser, a binary pump, an autosampler, and a thermostated column compartment was used for the analysis. Chromatographic separations were carried out on an Agilent Eclipse XDB-C18 column $(4.6 \mathrm{~mm} \times 250 \mathrm{~mm}, 5 \mu \mathrm{m})$ with $0.1 \%$ formic acid (as Solvent A) and acetonitrile (as Solvent B) at a flow rate of $1.0 \mathrm{~mL} / \mathrm{min}$ at room temperature. A linear gradient elution was applied from $15 \%-20 \% \mathrm{~B}$ at $0-10 \mathrm{~min}$, $20 \% \mathrm{~B}$ at $10-20 \mathrm{~min}, 20 \%-34 \% \mathrm{~B}$ at $20-45 \mathrm{~min}, 34 \%-48 \% \mathrm{~B}$ at $45-55 \mathrm{~min}, 48 \%-65 \% \mathrm{~B}$ at $55-70 \mathrm{~min}$, and $65 \%-80 \% \mathrm{~B}$ at $70-80 \mathrm{~min}$, and the equilibration time of gradient elution was $10 \mathrm{~min}$. Ten $\mu \mathrm{L}$ of the samples (after filtration with a $0.45 \mu \mathrm{m}$ Millipore filter) were injected, and signals were detected at $280 \mathrm{~nm}$ with UV detection. A standardized AR extract by calibrating different chemical markers was established. For biological analysis, the dried standardized extract was dissolved in phosphate-buffered saline and filtered through a $0.22 \mu \mathrm{m}$ filter before use. The pure flavonoids were dissolved by dimethyl sulfoxide before use.

2.3. DNA Transfection in Culture. Human embryonic kidney (HEK) 293T fibroblast cell is an excellent in vitro model in studying the physiological regulation of EPO expression, which is sensitive to hypoxia stress [7]. HEK 293T cell line was obtained from the American Type Culture Collection (ATCC, Manassas, VA) and cultured according to previous reports [11]. The HRE (5'-TCG AGG CCC TAC GTG CTG TCT CAC ACA GCC TGT CTG ACG-3') derived from human EPO gene contains a highly conserved HIF-1 binding site $\left(5^{\prime}\right.$-TAC GTG- $\left.3^{\prime}\right)$ and other unique cis-acting sequences $\left(5^{\prime}\right.$-CAC AG- $\left.3^{\prime}\right)$ that are functionally essential for hypoxic induction [11, 19]. Six HREs were synthesized, concatemerized, and then cloned in tandem (head-to-tail orientation) into pBI-GL vectors (BD Biosciences Clontech, San Jose, CA) that had a downstream reporter of firefly luciferase gene [11]. This vector was named as pHRE-Luc [19]. Cultured HEK293T cells were transiently transfected with pHRE-Luc by the calcium phosphate precipitation method [20]. The transfection efficiency was over $80 \%$, as determined by another control plasmid of having a $\beta$-galactosidase cDNA, under a cytomegalovirus enhancer promoter. The treatment of flavonoids, or AR extract, was done on transfected HEK293T cells. After 2 days, the cell lysates were collected for luciferase assay. In order to validate the response of the pHRE-Luc in transfected HEK293T cells, the cultures were exposed to hypoxia, serving as a positive control. The authentication of 
pHRE-Luc was confirmed by its activation in exposing to mineral oil layering and application of $\mathrm{CoCl}_{2}$ at $50 \mathrm{mM}$, and both methods were frequently used to mimic the effect of hypoxia [11].

2.4. Luciferase and Other Assays. The luciferase assay was performed by a commercial kit (Tropix Inc., Bedford, MA). In brief, cultures were lysed by a buffer containing $100 \mathrm{mM}$ potassium phosphate buffer ( $\mathrm{pH} 7.8$ ), 0.2\% TritonX-100, and $1 \mathrm{mM}$ dithiothreitol. The cell lysate was centrifuged in $13,200 \mathrm{rpm}(16,000 \times \mathrm{g})$ for $5 \mathrm{~min}$ in $4^{\circ} \mathrm{C}$, and $50 \mu \mathrm{L}$ of the supernatant was transferred to the assay plate and set on the luminance reading machine (FLUOstar OPTIMA, BMG, Germany). The readings of luminance intensity were equalized by the protein concentration of lysates, and the data indicated to the luciferase activities of the samples. Protein concentrations were measured routinely by Bradford's method with a kit from Bio-Rad Laboratories (Hercules, CA).

\section{Results}

3.1. $A R$ and Flavonoids Induce HRE-Mediated Transcriptional Activity. Calycosin, calycosin-7-O- $\beta$-D-glucoside, formononetin, and ononin are the major flavonoids contained within AR water extract (Figure 1(a)), which have inductive effect in EPO expression [10, 11]. HPLC analysis indicated that a standardized AR extract should contain the following marker compounds ( $\mu \mathrm{g} / 1 \mathrm{~g}$; mean $\pm \mathrm{SD}, n=4$ ) (Figure 1(b)): calycosin $(212.69 \pm 21.1)$, calycosin-7-O- $\beta$ D-glucoside (238.4 \pm 19.5), formononetin (150.12 \pm 13.7$)$, and ononin $(85.66 \pm 8.4)$. We first examined the abilities of AR extract and the flavonoids in the stimulation of HRE mediated transcriptional activity. The AR extract and four flavonoids, formononetin, ononin, calycosin, and calycosin-7-O- $\beta$-D-glucoside, were applied onto pHRE-Luctransfected fibroblasts for two days. The authentication of pHRE-Luc was confirmed by its activation in exposing to mineral oil layering or $\mathrm{CoCl}_{2}$ treatment, which was frequently used to mimic the effect of hypoxia [10]. Under the hypoxia by oil layering or $\mathrm{CoCl}_{2}$, the expression of pHRE-Luc was robustly induced in a time-dependent manner (Figure 2(a)). The AR extract and flavonoids showed significant induction on the pHRE-Luc activity in a dose-dependent manner (Figures 2(b) and 2(c)). The maximal induction by AR extract was over $70 \%$ of increase as compared to the background. Formononetin was the most potent flavonoid in the HRE activation, which induced a maximum over $90 \%$ at $1 \mu \mathrm{M}$, and the $\mathrm{EC}_{50}$ value was $\sim 0.05 \mu \mathrm{M}$. The $\mathrm{EC}_{50}$ of ononin, calycosin, and calycosin-7-O- $\beta$-D-glucoside were $0.56,0.66$, and $1.47 \mu \mathrm{M}$, and maximal inductions were $80 \%, 83 \%$, and $70 \%$ of increase, respectively (Figure 2(c)).

3.2. Optimization Strategy with Feedback System Control (FSC) Scheme. The FSC scheme consists of an iterative closed-loop of three operations, including formation of drug combinations, experimental readouts, and search algorithm (Figure 3). As the trials, a group of drug combinations selected from the parametric search space were applied in cultured cells. Induced cellular activities served as the fitness in drug effect evaluation. Based on the cellular responses, the search algorithm linked the cellular readouts and the drug combinations and therefore generated new combinations for subsequent iteration of experimental tests.

The four flavonoids were mixed and dissolved in dimethyl sulfoxide as the trial combinations. Based on the dose response of individual flavonoids (as from Figure 2(c)), we determined six concentration levels $(0,0.016,0.08,0.4,2$, and $10 \mu \mathrm{M})$ of each flavonoid to fully cover the effective range. Flavonoid combinations composed a parametric search space of 1,296 possible trials. The number of possible combinations rapidly increased with the number of flavonoids and concentration levels, as in an exponential form. Six index numbers of $0,1,2,3,4,5$ were assigned for the concentration levels of 0 , $0.016,0.008,0.4,2,10 \mu \mathrm{M}$, respectively; these index numbers were used later in the differential evolution search algorithm.

The success of FSC scheme heavily relied on the cellular readouts that closely mimicked the desired biological activity and the proper controls to evaluate the effects of the drug combinations. Our goal was to search for potent drug combinations that could stimulate EPO expression, and thus we used the HRE-mediated transcriptional activity as the initial readout. Since HRE is located on the promoter of EPO gene, the induced HRE activity can subsequently trigger the transcription of EPO gene [19].

The search algorithm plays an important role in the FSC scheme, which determines the efficiency and accuracy of the exploration. We used the differential evolution algorithm to perform a parallel exploration in the chemical optimization, due to its easy operation and previous successful application $[16,21,22]$. The principle and detailed application of the differential evolution algorithm in our FSC scheme were introduced in the supporting information (Figure 1(S), Supporting information, see supplementary materials available online at http://dx.doi.org/10.1155/2013/541436). Parameters, including the number of population (NP) and crossover probability (CR), were modified to better fit our system.

\subsection{Optimization of Flavonoid Combination Using FSC. We} iteratively evaluated 60 trial combinations in five iterations, each with at least triplicated samples (Figure 4). To determine the collaborative role of four flavonoids on pHRE-Luctransfected HEK 293T cells, we randomly selected six different combinations of the four flavonoids as trial combinations in the first iteration, using a random number generator in MATLAB (MathWorks). The HRE-mediated transcriptional activities, induced by the combinations, were found to be higher than $130 \%$. The highest response obtained in the first iteration was $232 \%$ increase of the control, induced by the 6 th combination $(0.08,0.08,0.4,10 \mu \mathrm{M}$ for formononetin, ononin, calycosin and calycosin-7-O- $\beta$-D-glucoside, resp.). Comparing with AR extract and individual flavonoid, higher activities induced by the drug combinations suggested possible collaborative effects among the flavonoids.

In the following iterations, we then attempted to optimize the combinations of the four flavonoids. The trial combinations were generated by the differential evolution (DE) 
<smiles>COc1ccc(-c2coc3cc(O[C@@H]4O[C@H](CO)[C@@H](O)[C@H](O)[C@H]4O)ccc3c2=O)cc1O</smiles>

Calycosin-7-O- $\beta$-D-glucoside<smiles>COc1ccc(-c2coc3cc(O[C@@H]4OC(CO)[C@@H](O)[C@H](O)[C@H]4O)ccc3c2=O)cc1</smiles>

Ononin<smiles>COc1ccc(-c2coc3cc(O)ccc3c2=O)cc1O</smiles>

Calycosin<smiles>COc1ccc(-c2coc3cc(O)ccc3c2=O)cc1</smiles>

Formononetin

(a)

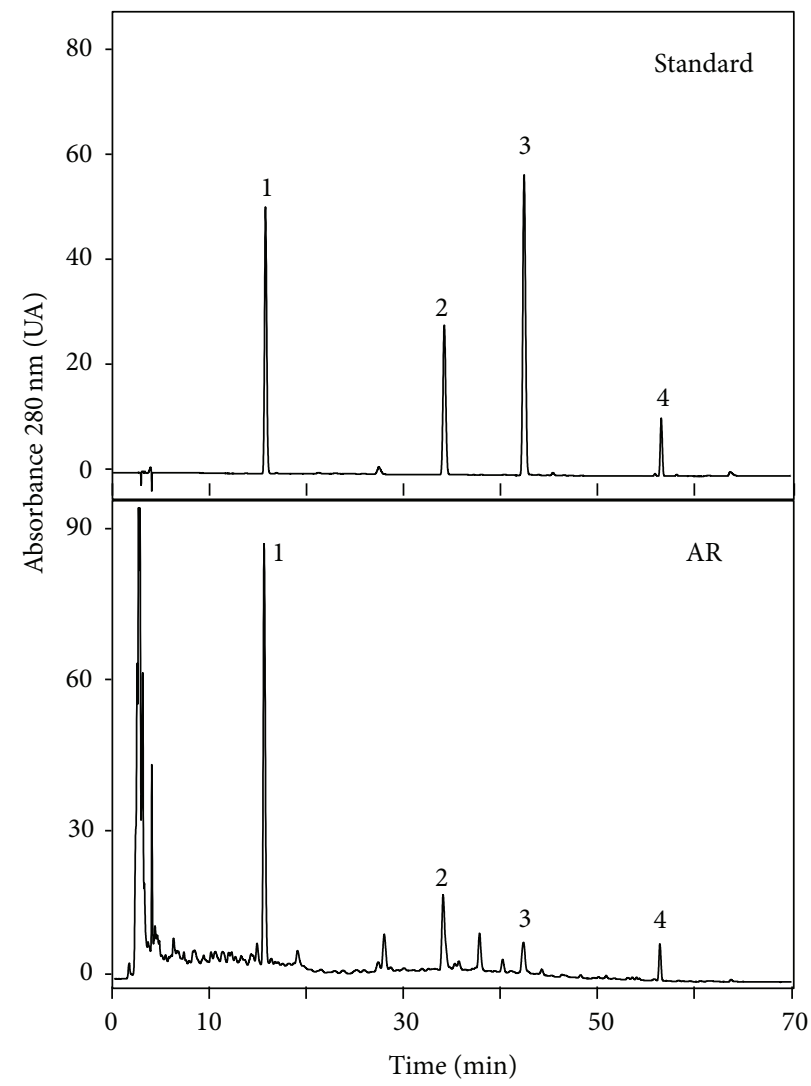

(b)

FIGURE 1: Chemical standardization of AR extract by HPLC fingerprint analysis. (a) Calycosin, calycosin-7-O- $\beta$-D-glucoside, formononetin, and ononin are the major flavonoids contained within AR water extract. (b) In a HPLC fingerprint at an absorbance of $280 \mathrm{~nm}$, the peaks corresponding to calycosin-7-O- $\beta$-D-glucoside (1), ononin (2), calycosin (3), and formononetin (4) were identified as standards (upper panel). The chemical amounts of these four compounds contained within the water extract of AR were calculated according to the HPLC results (lower panel). Representative chromatograms are shown; $n=3$. 
pHRE-Luc
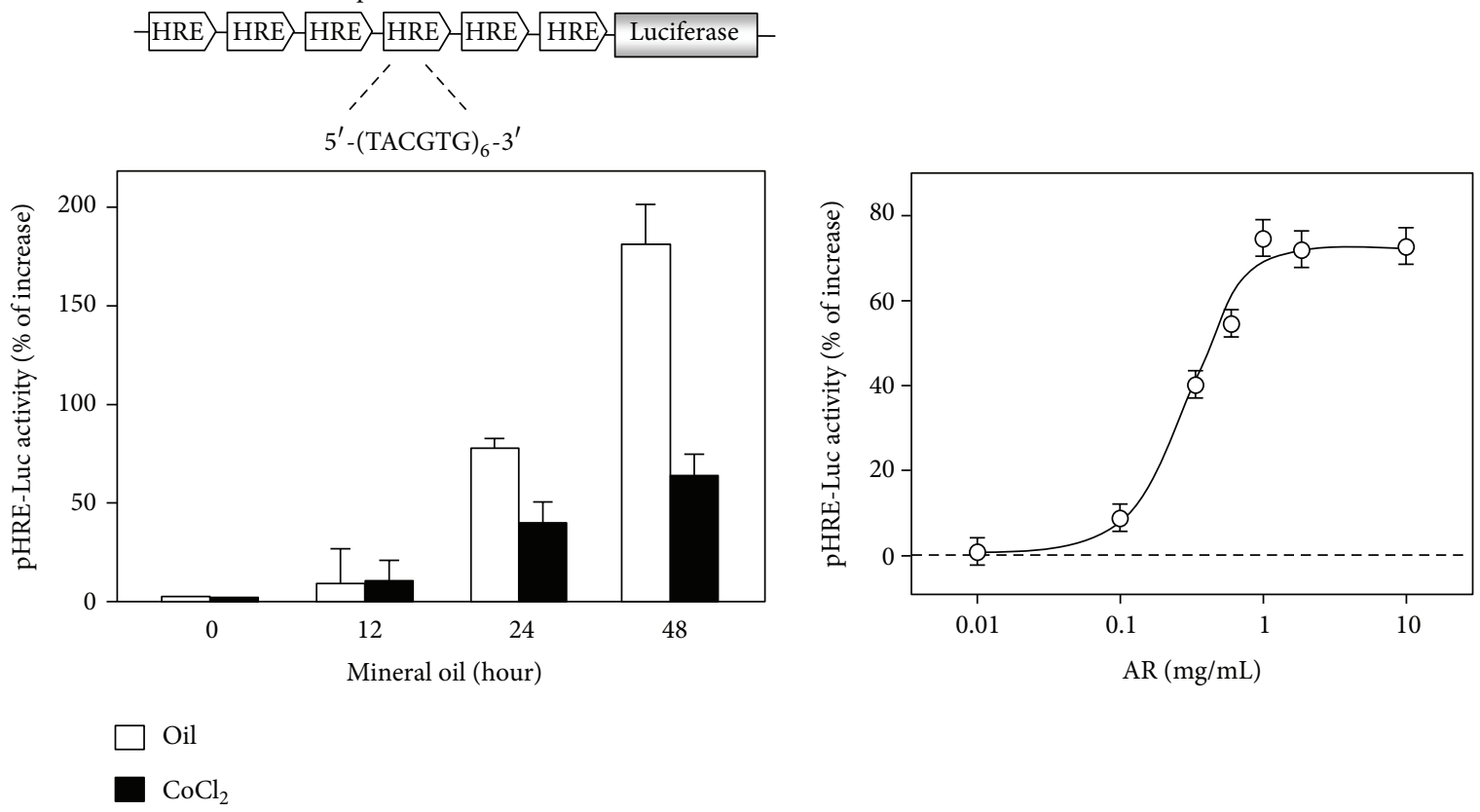

(a)

(b)

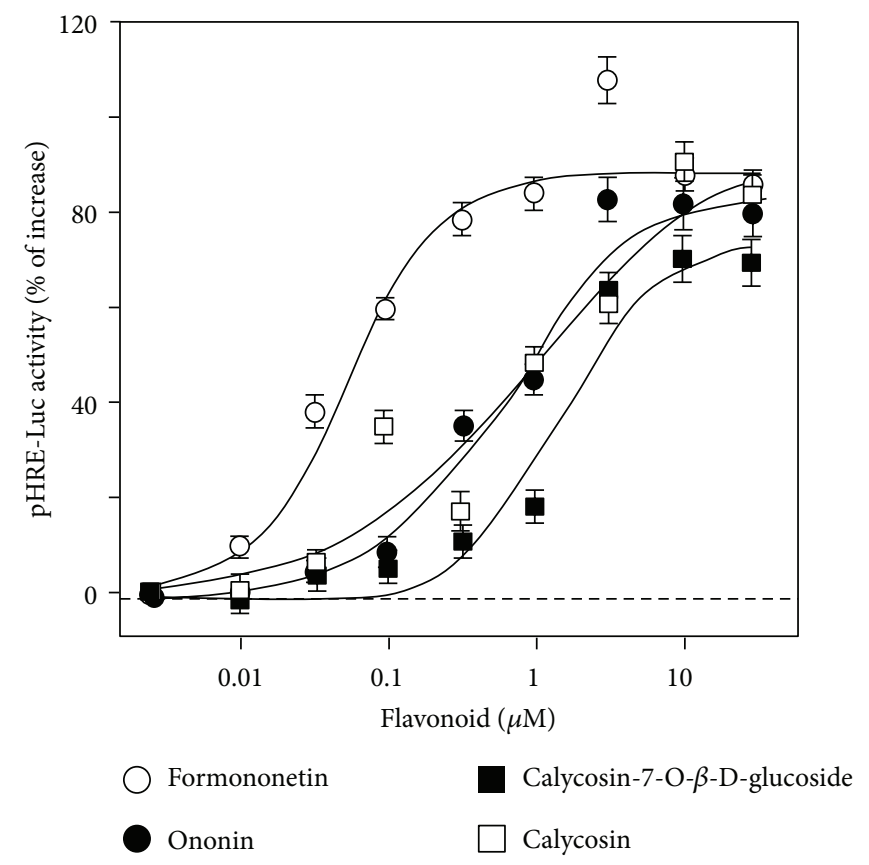

(c)

FIGURE 2: The AR extract and flavonoids stimulated the HRE-mediated transcriptional activity in cultured HEK293T cells. (a) Six repeats of hypoxia responsive elements (HRE: $5^{\prime}$-TCG AGG CCC TAC GTG CTG TCT CAC ACA GCC TGT CTG ACG-3') were subcloned in an expression vector of luciferase named as pHRE-Luc (upper panel). Cultured HEK293T cells, transfected with pHRE- Luc, were treated with $\mathrm{CoCl}_{2}(50 \mathrm{mM})$ or mineral oil layering for 0 to 48 hours. The cell lysates were subjected to luciferase assay to measure the transcriptional activity driven by HRE (lower panel). (b and c) The pHRE-Luc-transfected HEK293T cells were treated with AR extracts (b) and flavonoids (c) for 48 hours to determine the promoter-driven luciferase (pHRE-Luc) activity. Values are expressed as the percentage of increase to basal reading (untreated culture) and in mean $\pm \mathrm{SD}$, where $n=4$, each with triplicate samples. 


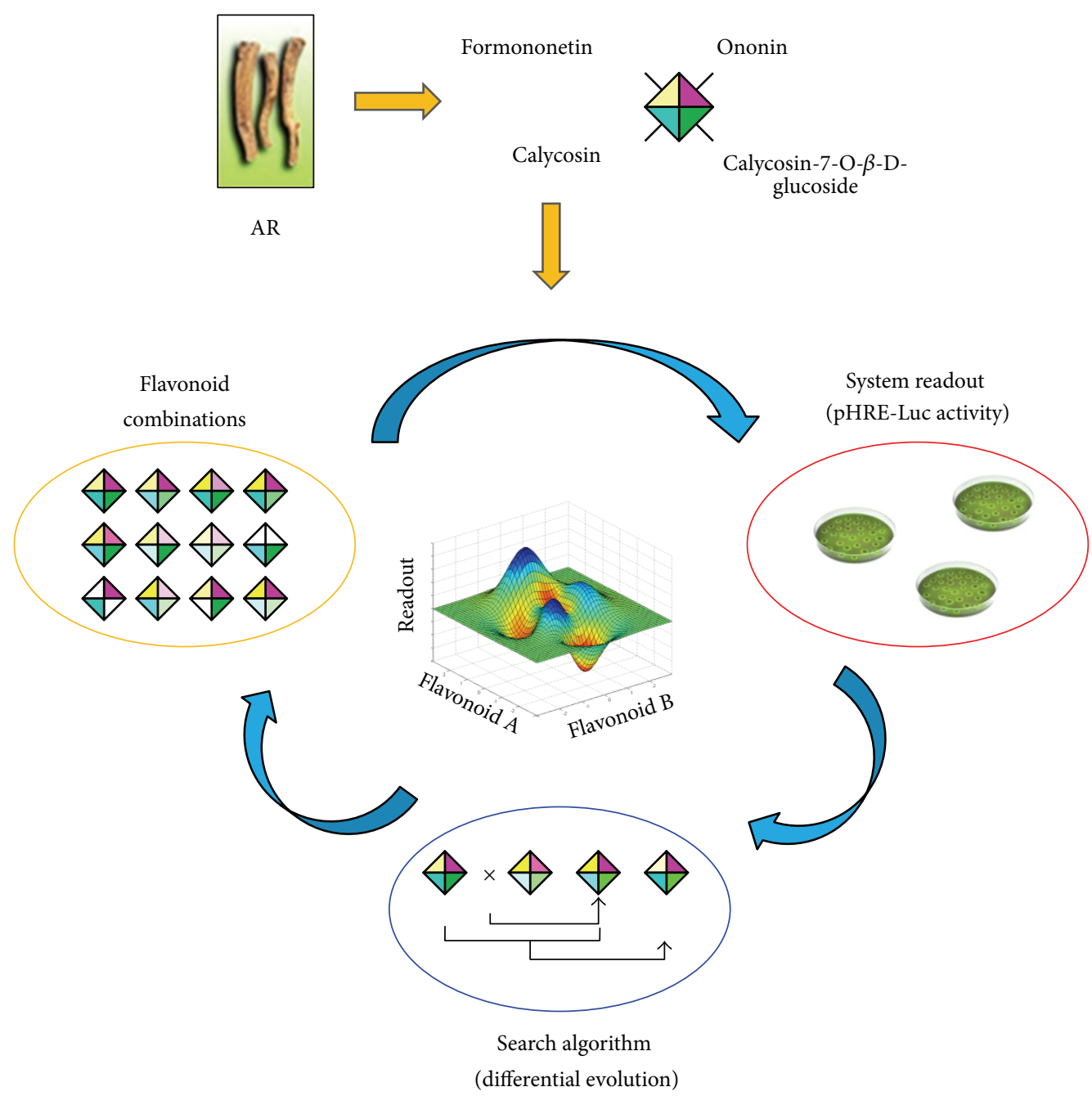

FIGURE 3: Optimization of the flavonoid combination by feedback system control (FSC) scheme. Feedback system control (FSC) scheme was used to optimize the flavonoid combinations. As trial combinations, the drug combinations of four flavonoids were applied onto pHRE-Luctransfected fibroblasts. The HRE-mediated transcriptional activity was used as the system readout to calculate the fitness of combinations. Differential evolution (DE) algorithm linked the drug combinations and the system readout as to generate new trial combinations in the next iteration.

algorithm, also using the MATLAB. To avoid being trapped in local maximum responses, the number of population was increased from 6 to 12, and finally 18 after iteration 3, and crossover probability was changed from 0.5 to 0.9 after iteration 2 . The FSC scheme iteratively updated the potent drug combinations towards better system performance, after the competition between original combinations and trial combinations in each iteration. (Figure 5). After the third iteration, by which we accumulatively tested 24 trials out of 1,296 possible combinations, we identified the 16th combination, that is, $0.08,0.08,0.4,0.08 \mu \mathrm{M}$ of the four flavonoids. This combination of flavonoids showed a $\sim 3$-fold improvement (333\%) in stimulating HRE-mediated transcriptional activity compared with individual flavonoids (see Figure 4). This unique combination was carried over through iterations 4 and 5 , showing promising drug potency.
Distinct effects of drug combinations in stimulating HRE-mediated transcriptional activity indicated the complicated reciprocity among flavonoids. Out of the 60 trials, we observed that 13 trial combinations induced lower HRE activation than AR and individual flavonoid. The minimum response was observed to be $39 \%$ at the 49 th combination, that is, $0.016,2,0.08,0.4 \mu \mathrm{M}$, which was $\sim 2$ fold decrease as compared with individual flavonoid (see Figure 4). Meanwhile, 16 potent combinations induced higher HRE-mediated transcriptional activity than $180 \%$ increase of control. Typical dosages of formononetin, ononin, calycosin and calycosin-7-O- $\beta$-D-glucoside in $1 \mathrm{mg} / \mathrm{mL}$ of AR extract were around $0.56 \mu \mathrm{M}, 0.16 \mu \mathrm{M}, 0.69 \mu \mathrm{M}$, and $0.513 \mu \mathrm{M}$, respectively [23-25]. Among the 16 potent combinations, the 16th $(0.08,0.08,0.4,0.08 \mu \mathrm{M})$ and 18th combinations $(0.016$, $0.4,0.08,0 \mu \mathrm{M})$ reduced the required dosage by $\sim 10$-fold 


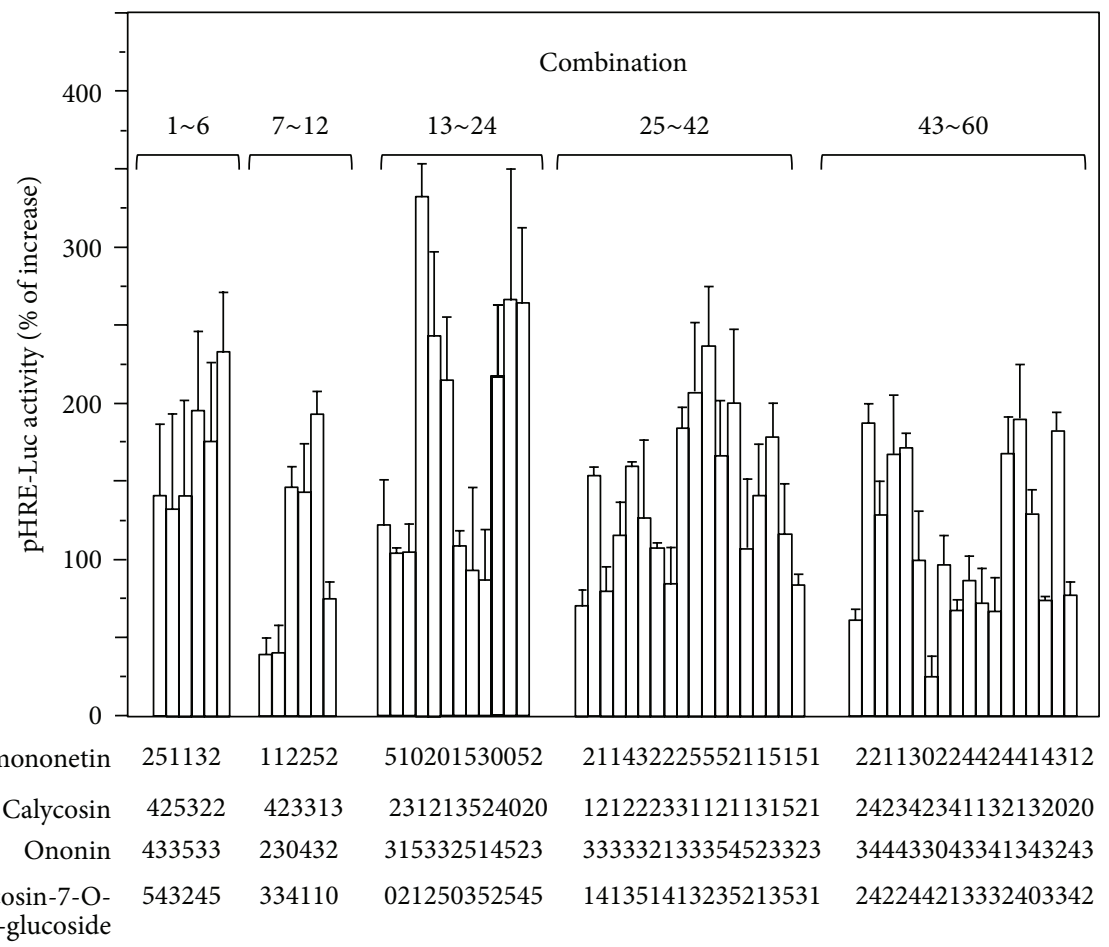

FIGURE 4: The HRE-mediated transcriptional activity induced by the 60 trial combinations in five iterations. The pHRE-Luc-transfected HEK293T cells were treated by the 60 trial combinations in five iterations, that is, $1-6,7-12,13-24,25-42$, and 43-60. The integer index of four flavonoids represented the corresponding concentration in each trial shown under each combination, that is, the index numbers of 0,1 , $2,3,4,5$ are corresponding to the final concentrations of $0,0.016,0.008,0.4,2,10 \mu \mathrm{M}$. After 48 hours, the promoter-driven luciferase (pHRELuc) activity was determined. The response to mineral oil layering, a positive control, was as effective as in Figure 2(a). Values are expressed as the percentage of increase to basal reading (untreated culture) and in mean $\pm \mathrm{SD}$, where $n=4$, each with triplicate samples.

compared with the amounts within $\mathrm{AR}$ water extract (see Figure 4).

The AR flavonoids possess a lot of biological functions as described previously [26]; however, the usage of combined flavonoids in a collaborative way is still challenging. To reveal if the flavonoids can work in a synergistic or antagonistic way in activating HRE mediated transcriptional activity, here we used the well-known median-effect equation proposed by Chou [27-30] (Figure 2(S), Supporting information). In Chou's theory, the combination index (CI) was used as the evaluation of synergistic effect. CI $<1$, CI $=1$, and CI $>1$ indicate synergism, additive effect, and antagonism, respectively. The CI values of the 60 trial combinations in our FSC optimization were quite different from each other, ranging from 0.033 to 28.5 , showing that the synergistic and antagonistic effects were closely related with the mixing ratio of flavonoids within the drug combinations (Figure 6). Interestingly, the minimum CI values were observed also in the 16th and 18th combinations, to be 0.033 and 0.044 , respectively. According to Chou's summary [30], the CI value smaller than 0.1 indicated possible very strong synergism among flavonoids to stimulate the HRE-mediated transcriptional activity, although dose-dependent studies of the combinations were required to obtain further information.

There is no guarantee that the combination we identified would be the best out of the 1,296 possible combinations, until we have exhaustively tested all the combinations. However, we can testify the effectiveness of this FSC scheme by simulation. Four different benchmark functions were used to mimic the biological system readout. The differential evolution algorithm was implemented exactly the same as used in our flavonoid optimization. Simulation results suggested that the FSC scheme was able to quickly pinpoint the optimal solution (Figure 3(S), Supporting information). Thus, our FSC optimization could be regarded as the stochastic optimal flavonoid combinations of the 1,296 possible combinations.

\section{Discussion}

The role of flavonoids in regulating the EPO expression has been known [10]. However, the detailed synergistic or antagonistic interaction among flavonoids has not been revealed yet, which therefore stimulates the engineering approach to optimize the flavonoid combinations by FSC scheme. By having FSC approach, we have saved over $90 \%$ of the laborious and time-consuming work that is required in exhaustively testing of all possible combinations. Two unique combinations, $(0.08,0.08,0.4,0.08) \mu \mathrm{M}$ and $(0.016,0.4,0.08$, 0) $\mu \mathrm{M}$ of formononetin, ononin, calycosin, and calycosin7-O- $\beta$-D-glucoside, were identified to be highly effective in stimulating HRE-mediated transcriptional activity, which 


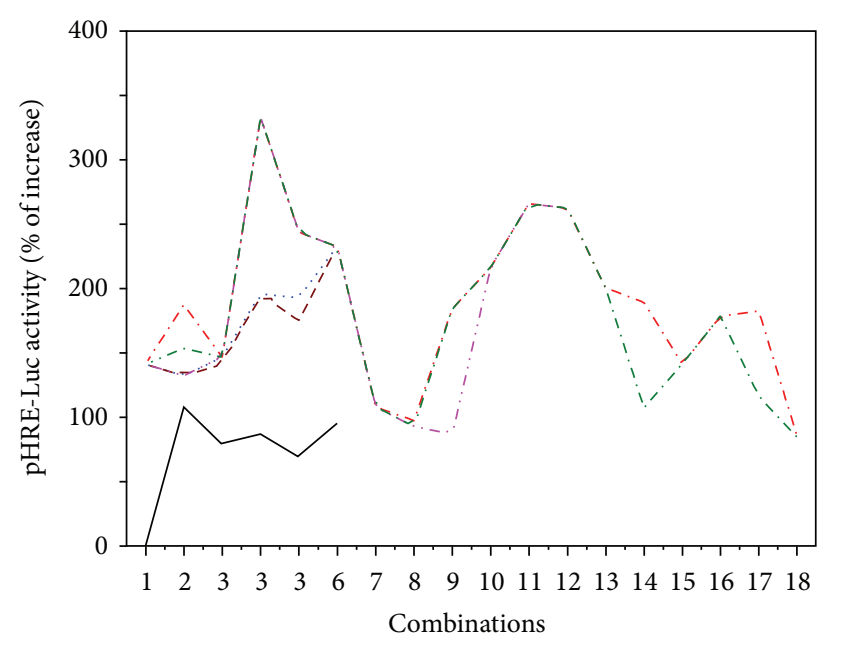

$\begin{array}{llll}- & \text { Initial } & -\cdots & \text { Iteration } 3 \\ --- & \text { Iteration } 1 & .-\cdot & \text { Iteration } 4 \\ \ldots \ldots & \text { Iteration } 2 & . \cdot- & \text { Iteration } 5\end{array}$

FIGURE 5: The HRE-mediated transcriptional activity induced by the winning population of flavonoid combinations in each iteration In each iteration, the $x$-axis represents the $i$ th combination in current winning population. Winning combinations are determined after competition between original combinations and trial combinations, as in Figure 1(S). Values are expressed as the mean value in Figure 4. Initial combinations are comprised of control, AR extract, and individual flavonoid. The FSC scheme iteratively drove combinations in the population towards higher HRE-mediated transcriptional activity; that is, the best response of $3 \mathrm{rd}$ combination in the population was found in iteration 3 , and carried over through iterations 4 and 5 .

was increased $\sim 3$-fold by the two optimized combinations, while the AR herbal extract and individual flavonoid can only induce the transcriptional activity by about $80 \%$. The concentration of these four flavonoids that we used here is much lower than that in the herbal extract of AR. Not only in cultured cells, our preliminary results indicated that the application of optimized combination of flavonoids could achieve maximal activation of red blood cell production in animal studies (Zhang et al., unpublished result). By using the FSC scheme as described here, the transcriptional activity of EPO was greatly increased, and the amount of flavonoids used was reduced by $\sim 10$-fold.

The successful application of FSC scheme in optimizing the combination of four flavonoids provides hints in study of TCM formulae. According to TCM theory, the herbal formulae should be prepared in a unique methodology having specific combination of different herbs as a formula (named as $\mathrm{Fu}$ Fang). In general, the combination amongst different herbs within a decoction will directly affect the pharmacological properties of a herbal formula. Indeed, our previous work has supported the usage of the best combination of two herbs in Danggui Buxue Tang, a traditional herbal decoction. Having the best combination of herbs, this herbal decoction possesses enriched chemical and biological properties [23]. However,

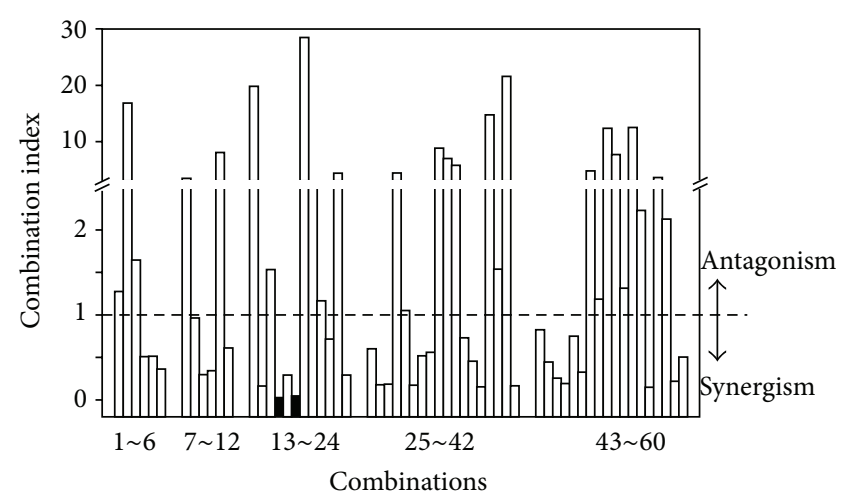

Figure 6: Combination index of the 60 trial combinations. The combination indexes (CIs) $<1,=1$, and $>1$ indicate synergism, additive effect, and antagonism, respectively. The calculation of CI was accord with the theory of Chou. Sixty combinations out of 5 five iterations are shown here. The 16th and 18th combinations (black bars) showed the strongest synergism, that is, the smallest CI value.

the major problem in the optimization of herbal mixtures is the number of possible combinations. For example, over thousands of possible herb combinations could be found even in a herbal decoction having two herbs. Experimentally, it is impossible to try all these combinations of herbal extracts in optimizing a TCM formula. Having FSC scheme, only about tens of trials are required to optimize the combination, and almost $90 \%$ of the work can be trimmed. Thus, the FSC scheme as described here can be used as a new approach to optimize the combination of herbal extracts in TCM formula, which will provide more evidence for the better use of herbal mixture. Moreover, the FSC scheme may be further developed to predict the drug combinations [31].

\section{Conclusions}

In summary, it can be stated that optimized combinations of flavonoids have a strong HRE-mediated transcriptional activity, which suggests that the optimized flavonoids could have good activity in stimulating the regulatory element of erythropoietin at a very low concentration. The application of FSC scheme could be able to identify potent drug combination of ingredients within herbal medicines, as well as in future its application in finding optimized combination of Chinese herbal mixture.

\section{Authors' Contribution}

H. Yu and W. L. Zhang contributed equally to this work.

\section{Conflict of Interest}

The authors have no financial or personal relationships with other people or organizations that can inappropriately influence the work presented in the paper. 


\section{Acknowledgments}

This research was supported by grants from Croucher Foundation (CAS-CF07/08.SC03) to K. W. K. Tsim and a seed grant from HKUST (PROVOST12EG01) to Y. K. Lee.

\section{References}

[1] Y. C. Liu, K. Vian, P. Eckman, T. Y. Fang, and L. D. Chen, The Essential Book of Traditional Chinese Medicine: Theory, Clinical Practice, Columbia University Press, New York, NY, USA, 1988.

[2] T. Xue and R. Roy, "Studying traditional Chinese medicine," Science, vol. 300, no. 5620, pp. 740-741, 2003.

[3] D. Normile, "The new face of traditional Chinese medicine," Science, vol. 299, no. 5604, pp. 188-190, 2003.

[4] X. Q. Ma, Q. Shi, J. A. Duan, T. T. X. Dong, and K. W. K. Tsim, "Chemical analysis of Radix Astragali (Huangqi) in China: a comparison with its adulterants and seasonal variations," Journal of Agricultural and Food Chemistry, vol. 50, no. 17, pp. 4861-4866, 2002.

[5] J. L. Ríos and P. G. Waterman, "A review of the pharmacology and toxicology of astragalus," Phytotherapy Research, vol. 11, no. 6, pp. 411-418, 1997.

[6] L. W. Qi, Q. T. Yu, P. Li et al., "Quality evaluation of Radix Astragali through a simultaneous determination of six major active isoflavonoids and four main saponins by high-performance liquid chromatography coupled with diode array and evaporative light scattering detectors," Journal of Chromatography A, vol. 1134, no. 1-2, pp. 162-169, 2006.

[7] C. Dame, K. M. Kirschner, K. V. Bartz, T. Wallach, C. S. Hussels, and H. Scholz, "Wilms tumor suppressor, Wt1, is a transcriptional activator of the erythropoietin gene," Blood, vol. 107, no. 11, pp. 4282-4290, 2006.

[8] W. Jelkmann, "Erythropoietin: structure, control of production, and function," Physiological Reviews, vol. 72, no. 2, pp. 449-489, 1992.

[9] P. Maxwell, "HIF-1: an oxygen response system with special relevance to the kidney," Journal of the American Society of Nephrology, vol. 14, no. 11, pp. 2712-2722, 2003.

[10] K. Y. Z. Zheng, R. C. Y. Choi, A. W. H. Cheung et al., "Flavonoids from radix astragali induce the expression of erythropoietin in cultured cells: a signaling mediated via the accumulation of hypoxia-inducible factor-1 $\alpha$," Journal of Agricultural and Food Chemistry, vol. 59, no. 5, pp. 1697-1704, 2011.

[11] K. Y. Z. Zheng, R. C. Y. Choi, H. Q. H. Xie et al., “The expression of erythropoietin triggered by Danggui Buxue Tang, a Chinese herbal decoction prepared from Radix Astragali and Radix Angelicae Sinensis, is mediated by the hypoxia-inducible factor in cultured HEK293T cells," Journal of Ethnopharmacology, vol. 132, no. 1, pp. 259-267, 2010.

[12] J. E. Dancey and H. X. Chen, "Strategies for optimizing combinations of molecularly targeted anticancer agents," Nature Reviews Drug Discovery, vol. 5, no. 8, pp. 649-659, 2006.

[13] J. D. Feala, J. Cortes, P. M. Duxbury, C. Piermarocchi, A. D. McCulloch, and G. Paternostro, "Systems approaches and algorithms for discovery of combinatorial therapies," Wiley Interdisciplinary Reviews, vol. 2, no. 2, pp. 181-193, 2010.

[14] T. Efferth, P. C. H. Li, V. S. B. Konkimalla, and B. Kaina, "From traditional Chinese medicine to rational cancer therapy," Trends in Molecular Medicine, vol. 13, no. 8, pp. 353-361, 2007.
[15] S. Li, B. Zhang, and N. Zhang, "Network target for screening synergistic drug combinations with application to traditional Chinese medicine," BMC Systems Biolology, vol. 5, supplement 1, p. S10, 2011.

[16] H. Yan, B. Zhang, S. Li, and Q. Zhao, "A formal model for analyzing drug combination effects and its application in TNFalpha-induced NFkappaB pathway," BMC Systems Biolology, vol. 4, article 50, 2010.

[17] P. K. Wong, F. Yu, A. Shahangian, G. Cheng, R. Sun, and C. M. Ho, "Closed-loop control of cellular functions using combinatory drugs guided by a stochastic search algorithm," Proceedings of the National Academy of Sciences of the United States of America, vol. 105, no. 13, pp. 5105-5110, 2008.

[18] H. Tsutsui, B. Valamehr, A. Hindoyan et al., "An optimized small molecule inhibitor cocktail supports long-term maintenance of human embryonic stem cells," Nature Communications, vol. 2, no. 1, article 167, 2011.

[19] D. E. Post and E. G. Van Meir, "Generation of bidirectional hypoxia/HIF-responsive expression vectors to target gene expression to hypoxic cells," Gene Therapy, vol. 8, no. 23, pp. 1801-1807, 2001.

[20] H. Q. Xie, R. C. Y. Choi, K. W. Leung et al., "Regulation of a transcript encoding the proline-rich membrane anchor of globular muscle acetylcholinesterase: the suppressive roles of myogenesis and innervating nerves," The Journal of Biological Chemistry, vol. 282, no. 16, pp. 11765-11775, 2007.

[21] R. Storn and K. Price, "Differential evolution-a simple and efficient heuristic for global optimization over continuous spaces," Journal of Global Optimization, vol. 11, no. 4, pp. 341359, 1997.

[22] C. P. Sun, T. Usui, F. Yu et al., "Integrative systems control approach for reactivating Kaposi's sarcoma-associated herpesvirus (KSHV) with combinatory drugs," Integrative Biology, vol. 1, no. 1, pp. 123-130, 2009.

[23] T. T. X. Dong, K. J. Zhao, Q. T. Gao et al., "Chemical and biological assessment of a Chinese herbal decoction containing Radix Astragali and Radix Angelicae Sinensis: determination of drug ratio in having optimized properties," Journal of Agricultural and Food Chemistry, vol. 54, no. 7, pp. 2767-2774, 2006.

[24] Q. Gao, J. Li, J. K. H. Cheung et al., "Verification of the formulation and efficacy of Danggui Buxue Tang (a decoction of Radix Astragali and Radix Angelicae Sinensis): an exemplifying systematic approach to revealing the complexity of Chinese herbal medicine formulae," Chinese Medicine, vol. 2, article 12, 2007.

[25] R. C. Choi, Q. T. Gao, A. W. Cheung et al., "A Chinese herbal decoction, Danggui Buxue Tang, stimulates proliferation, differentiation and gene expression of cultured osteosarcoma cells: genomic approach to reveal specific gene activation," Evidence-Based Complementary and Alternative Medicine, vol. 2011, Article ID 307548, 13 pages, 2011.

[26] H. Wagner, R. Bauer, P. G. Xiao, J. M. Chen, and G. Michler, "Chinese drug monographs and analysis: Radix Astragali (Huangqi)," in Verlag für Ganzheitliche Medizin Dr. Erich Wühr GmbH, A. Staudinger, Ed., Kötzting/Bayer, Wald, Germany, 1997.

[27] T. C. Chou, "The median-effect principle and the combination index for quantitation of synergism and antagonism," in Synergism and Antagonism in Chemotherapy, Academic Press, San Diego, Calif, USA, 1991.

[28] T. C. Chou, "Theoretical basis, experimental design, and computerized simulation of synergism and antagonism in drug 
combination studies," Pharmacological Reviews, vol. 58, no. 3, pp. 621-681, 2006.

[29] T. C. Chou, "The mass-action law based algorithms for quantitative econo-green bio-research," Integrative Biology, vol. 3, no. 5, pp. 548-559, 2011.

[30] T. C. Chou, "The mass-action law based algorithm for costeffective approach for cancer drug discovery and development," American Journal of Cancer Research, vol. 1, no. 7, pp. 925-954, 2011.

[31] X. M. Zhao, M. Iskar, G. Zeller, M. Kuhn, V. van Noort, and P. Bork, "Prediction of drug combinations by integrating molecular and pharmacological data," PLoS Computational Biology, vol. 7, no. 12, Article ID e1002323, 2011. 


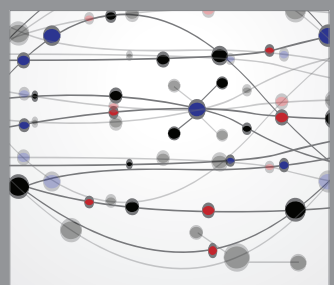

The Scientific World Journal
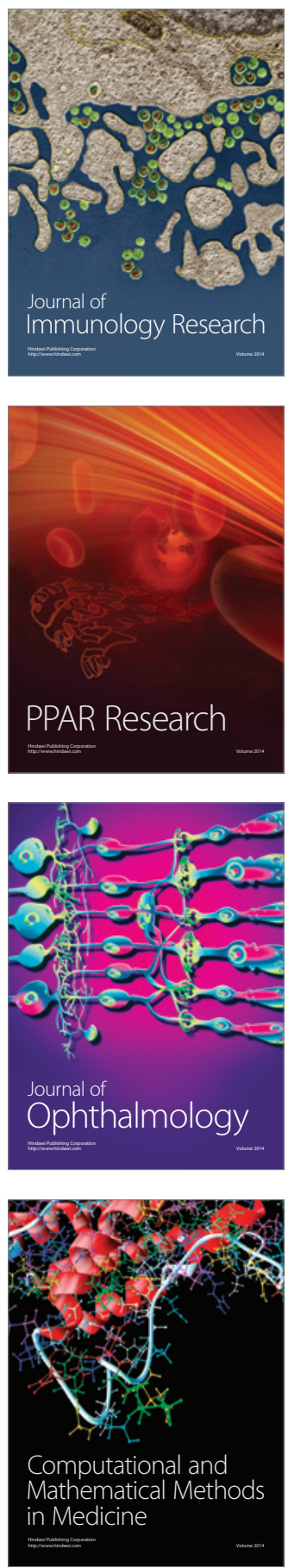

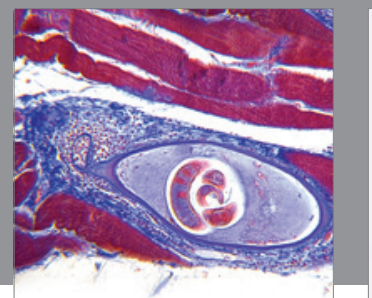

Gastroenterology

Research and Practice
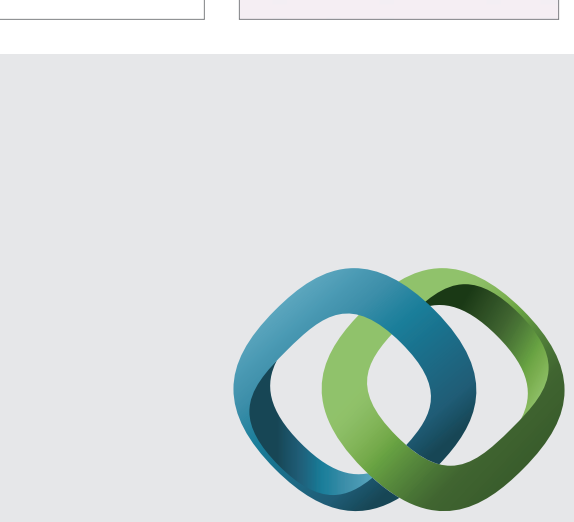

\section{Hindawi}

Submit your manuscripts at

http://www.hindawi.com
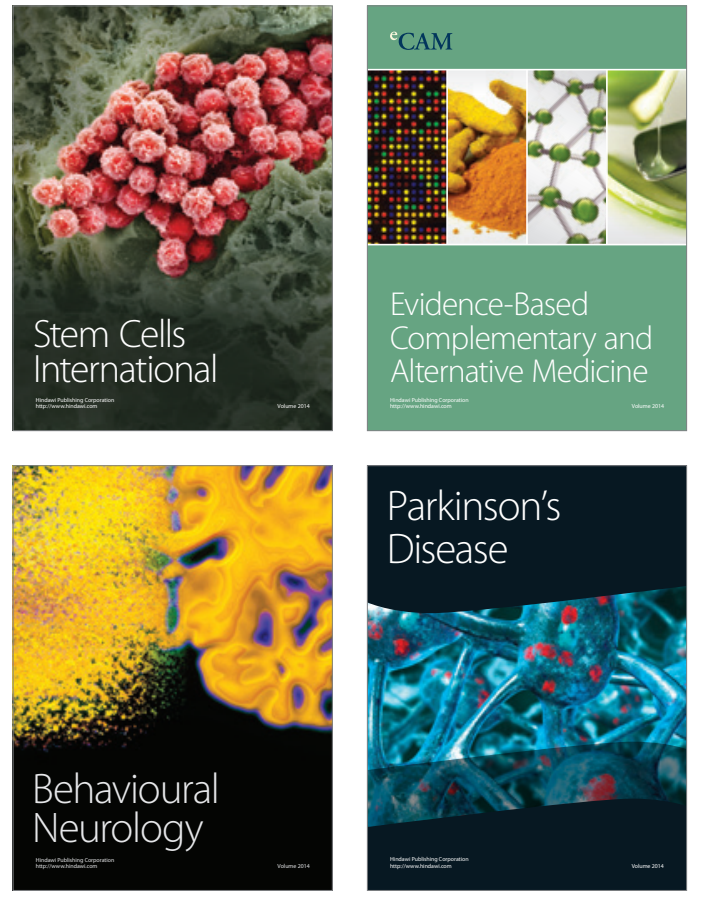
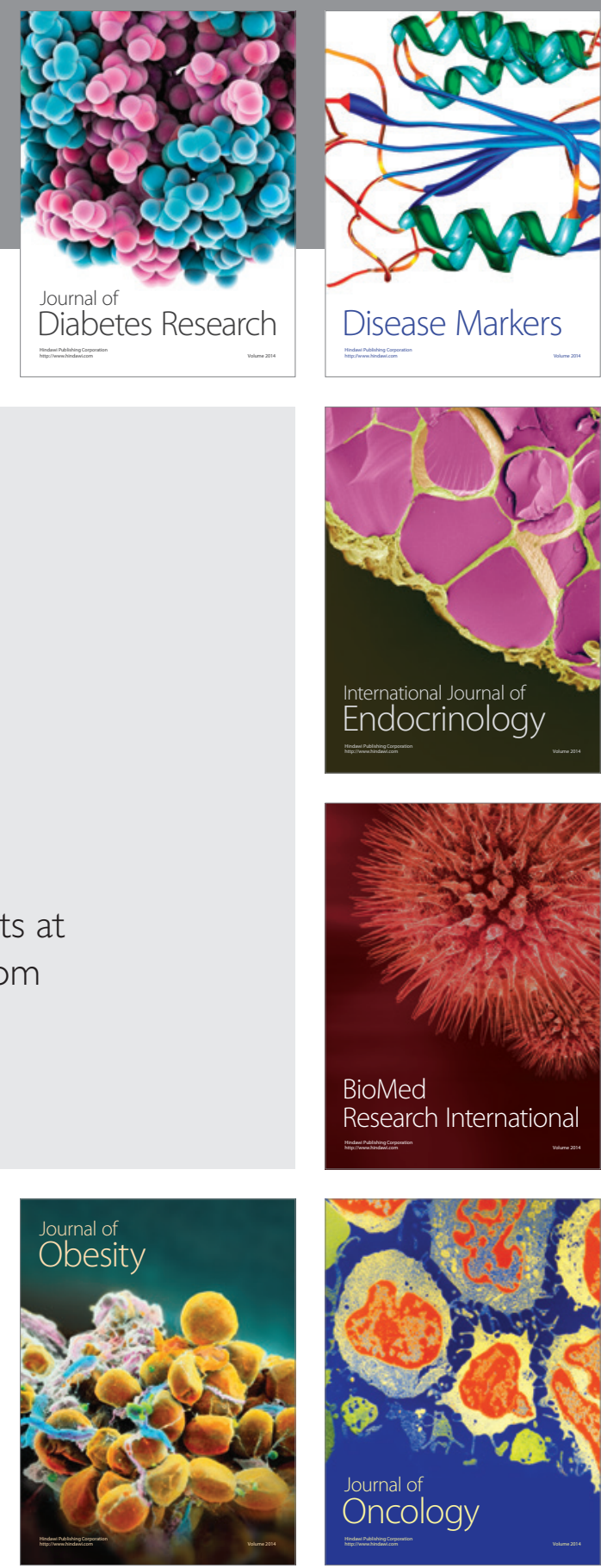

Disease Markers
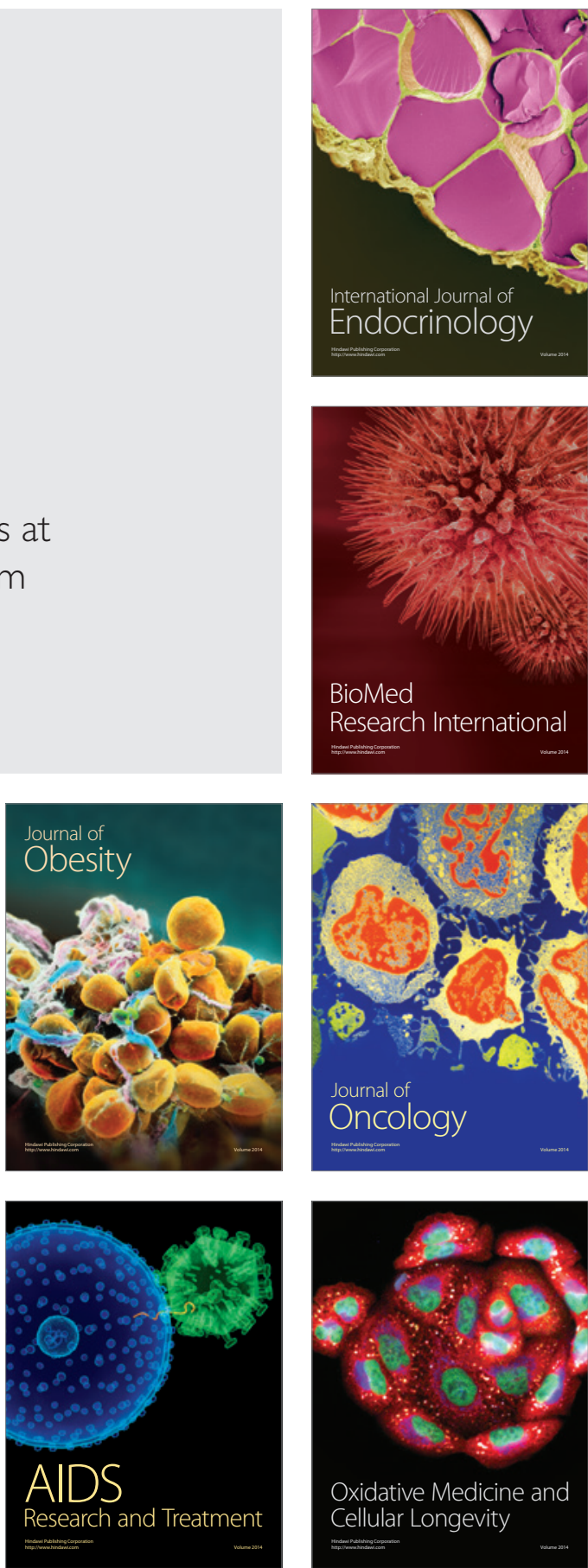